\title{
THROUGHPUT ANALYSIS OF AGGREGATION WITH FRAGMENT RETRANSMISSION PLUS QUALITY OF SERVICE SCHEME
}

\author{
${ }^{1}$ Teuku Yuliar Arif and ${ }^{2}$ Riri Fitri Sari \\ ${ }^{1}$ Department of Electrical Engineering, \\ Faculty of Engineering, Syiah Kuala University, Banda Aceh, Indonesia \\ ${ }^{2}$ Department of Electrical Engineering, \\ Faculty of Engineering, University of Indonesia, Depok, Indonesia
}

Received 2013-02-15, Revised 2013-07-08; Accepted 2013-08-28

\begin{abstract}
Aggregation with Fragment Retransmission (AFR) is a scheme designed for frame transmission to increase efficiency at the MAC layer throughput. AFR provides a higher throughput compare to Distributed Coordination Function (DCF) scheme. However AFR scheme did not has QoS service function when compared to DCF scheme. DCF scheme already has contention-based QoS service using Enhanced Distributed Coordination Function (EDCA) scheme. In this study, we enhanced the AFR scheme in order to provide the QoS function and called it as AFR plus QoS or abbreviated as AFR+Q. We developed the analytical model to investigate the saturation throughput of AFR+Q scheme. We designed the Markov chain model as state transition of the backoff counter from each Access Category (AC) and we derived the mathematical model of the transmission probability and the saturation throughput of each AC. The simulation result using Matlab shown that the AFR+Q scheme provide higher saturation throughput compared to EDCA scheme. The higher throughput in AFR+Q scheme is produced by the better transmission probability, employed packet fragmentation and aggregate frame schemes.
\end{abstract}

Keywords: IEEE 802.11n, Aggregation with Fragment Retransmission (AFR), Enhanced Distributed Channel Access (EDCA), Aggregation with Fragment Retransmission Plus QoS (AFR+Q), Throughput

\section{INTRODUCTION}

The first standard of IEEE 802.11 Wireless Local Area Network (WLAN) ratified in 1999 was only with 1 Mbps of Pysical (PHY) layer rate (IEEE Std. 802.11, 1999). Today the maximum rate increases up to 600 Mbps using the latest PHY layer capability, i.e., Multiple Input Multiple Output (MIMO) and Orthogonal Frequency Division Multiplex (OFDM) as described in IEEE $802.11 \mathrm{n}$ standard. This new standard was ratified in late 2009 (IEEE Std. 802.11e, 2009). The high data rate speed provided by IEEE 802.n standard can be used for transmitting high quality multimedia traffic that requires very high bit rate, e.g., High Definition Television (HDTV) and 3D video. The problems occur when the physical layer is in a heavy load condition, because some traffic categories e.g., voice and video, need a stable transfer rate and delay guarantee. Hence, to guarantee the Quality of Service (QoS) for the data traffics, the QoS function is an essential requirement in very high speed WLAN.

Referring to the standard of IEEE 802.11(IEEE Std. 802.11, 1999), each packet will be treated equally without channel access priority by Medium Access Control (MAC) layer using Distributed Coordination Function (DCF) scheme when transmitted. In this

Corresponding Author: Teuku Yuliar Arif, Department of Electrical Engineering, Faculty of Engineering, Syiah Kuala University, Banda Aceh, Indonesia 
respect, either video or data packet will be treated with equal channel access probability when transmitted. To resolve the problem, IEEE sets a standard 802.11e (IEEE Std. 802.11e, 2005), which is intended to provide QoS fuction on MAC layer based on channel access priority. There are two different schemes of channel access priority on IEEE 802.11e, i.e., Enhanced Distributed Channel Access (EDCA) which is based on contention and HCF Controlled Channel Access (HCCA) which is non contention.

Aggregation with Fragment Retransmission (AFR) is a MAC protocol scheme that improves DCF performance. It was introduced by (Li et al., 2009) and initially proposed in the IEEE $802.11 \mathrm{n}$ task group. In AFR scheme, a number of packets will be aggregated to become a large frame before transmission. Before aggregated, each packet will be fragmented and a Frame Check Sequence (FCS) will be added between the two fragments. In the receiver side, if an error occurs then only one fragment will be retransmitted. This mechanism can improve the efficiency and the saturation throughput of MAC layer with different wireless channel Bit Error Rate (BER) significantly.

Recently, there are several publications in the development of AFR scheme. Olteanu and Xiao (2010), a security function was added in AFR scheme and the impact to AFR performance was described. However, the works in (Olteanu and Xiao, 2010) did not consider QoS function, e.g., contention based QoS which is used in IEEE 802.11e EDCA. In other words, when both IEEE 802.11n and IEEE 802.11e are adopted, the performance analysis of QoS with aggregation and fragmentation mechanism must be considered. In this work, we add QoS function in the AFR scheme and analyze its performance. Throughout the rest of the paper, we use AFR plus QoS (AFR+Q) terminology to refer to the AFR scheme that has been enhanced with a QoS function.

The remainder of the paper is organized as follows. Section II reviews the state of the art. Section III describes the related work. Section IV shows the analytical model for AFR+Q. Section V verifies the performance of AFR+Q using simulation tool. Finally, Section VI concludes the paper by summarizing results and outlining the future works.

\section{STATE OF THE ART}

In this section, we present the state of the art the area of contention based QoS with aggregation and fragmentation consideration. After presenting briefly the
DCF, EDCA and AFR scheme, we introduces the AFR+Q scheme.

\subsection{IEEE 802.11 DCF}

The IEEE 802.11 standard specifies two different channel access methods to use the wireless channel simultaneously between stations in WLAN. The first method is a centralized channel access control using a scheme called Point Coordination Function (PCF). The second method is a distributed channel access control using a scheme called Distributed Coordination Function (DCF) (IEEE Std. 802.11, 1999). DCF scheme manages the use of distributed channel by applying Carrier Sense Multiple Access with Collision Avoidance (CSMA/CA) algorithm. It is mostly used on the current WLAN devices.

Here we describe the mechanism how DCF scheme manages the use of channel simultaneously between stations in a WLAN. When the MAC layer of a station has packets to transmit, it performs a prior check to see if the channel is being used by another station using CSMA/CA algorithm. If the channel is idle-indicated by the appearance of the duration of DCF Inter-frame Space (DIFS), the packet is ready to be transmitted. The station then runs a backoff procedure by selecting the backoff counter's initial value uniformly in a range of $\left[0, \mathrm{CW}_{\mathrm{min}^{-}}\right.$ 1], where $\mathrm{CW}_{\min }$ is the minimum contention window size. Shortly after the DIFS period or at the beginning of the time slot, the backoff counter value starts to decrease but this will be suspended if the channel is found to be in a busy condition during the process. When the channel returns to idle condition, the decrement process will be continued. If the backoff counter reaches zero, then the packet will be transmitted. The receiver station sends an Acknowledgement (ACK) packet to the sender station for every packet successfully received.

The IEEE 802.11 standard also specifies two operational modes for transmitting packets on DCF scheme. In the first operational mode, the data packet is transmitted directly when the backoff counter has reached zero and the channel is in idle condition. This mode is called Basic Access mode. The sender station recognizes that there are no errors found on the packet transmitted if it has received ACK packet from the receiving station. While in the second operational mode, the station will send Request to Send (RTS) packet broadcast before transmitting the data packet. All other stations in WLAN coverage that receive RTS packet will postpone their transmission process as long as the time value of the RTS packet. The receiving station then transmits Clear to Send (CTS) packet to confirm the receipt of the RTS packet. 


\subsection{IEEE 802.11e EDCA}

DCF scheme does not have QoS function to provide packet transmission priority to each type of traffic as well as to every different station on WLAN. DCF scheme sets up a distributed channel access with equal probability between stations when transmitting a packet. IEEE 802.11e standard developed DFC scheme to provide QoS in MAC layer and called as Hybrid Coordination Function (HCF) (IEEE Std. 802.11e, 2005). HCF has two approaches of channel access method, i.e., Enhanced Distributed Channel Access (EDCA) and HCF controlled channel access (HCCA).

In order to provide service differentiation, there are four Access Category (AC) used to accommodate four different traffic classes on EDCA scheme. Each AC is has an index, for instance $z$ where $0 \leq \mathrm{z} \leq 3$. AC [0] has the highest channel access priority used to transmit voice packet (AC_VO), AC [1] for video packet (AC_V1), AC [2] for best effort packet (AC_BE) and the lowest access priority, $\mathrm{AC}$ [3] is used to transmit the background packet (AC_BK). Different channel access priority between $\mathrm{AC}[\mathrm{z}]$ is determined based on AIFS Number ( $\left.{ }^{\mathrm{AIFSN}}[\mathrm{AC}]\right)$ parameter, minimum $\left(\mathrm{CW}_{\min }[\mathrm{AC}]\right)$ and maximum $\left(\mathrm{CW}_{\max }[\mathrm{AC}]\right)$ contention window and Transmission Opportunity (TXOP). These parameters are also called as EDCA parameters set. Each packet received by MAC layer in EDCA scheme will be mapped in an AC according to priority information contained in the package.

\subsection{AFR MAC Protocol}

In DCF scheme, each packet is added MAC and PHY headers prior to its transmission. When the data rate of PHY layer continues to increase from $11 \mathrm{Mbps}$ to $600 \mathrm{Mbps}$ as defined in standard IEEE $802.11 \mathrm{~b} / \mathrm{a} / \mathrm{g} / \mathrm{n}$, the efficiency of MAC layer throughput also decreases. The decreasing of throughput efficiency is due to the increase of the data rate of PHY layer. This caused by the overhead of MAC and PHY layer is not decreased so it dominates the delivery time of the frame. In other words, the addition of MAC and PHY header on each packet transmitted when the data rate of PHY layer continues to increase causes the decreasing of MAC layer throughput.

Motivated by the inefficiency of DCF scheme, (Li et al., 2009) proposed the AFR scheme to improve the efficiency of MAC layer throughput by reducing the overhead of MAC and PHY layer when a number of packets are transmitted. In the AFR scheme, when MAC layer receives packets from the upper layer, those packets are aggregated into a large frame. Before aggregated, the size of the packet will be checked in advance and if the size of the packet exceeds the fragment threshold, the packet will be fragmented according to the fragment threshold (Li et al., 2006). Next, the frame containing a number of packet fragments will be transmitted as on DCF scheme. The difference between DCF and AFR scheme lies on how many packets can be transmitted by one MAC and PHY layer header. In DCF, one MAC and PHY layer header is used to transmit one packet only, while on AFR scheme, one MAC and PHY layer header is used to transmit several packets simultaneously.

\subsection{AFR+Q MAC Protocol}

AFR scheme can increase throughput efficiency of MAC layer significantly compared to DCF scheme (Li et al., 2009). However, QoS services for the packet transmitted using AFR scheme is not available yet. In this study, we propose a new scheme that was developed from AFR scheme by adding QoS function. We call this scheme as AFR plus QoS or abbreviated as AFR+Q. The QoS function that we added is based on contention, similar to EDCA scheme.

In AFR+Q scheme, there are four Access Category (AC) used to accommodate four different traffic classes as shown in Fig. 1. Each AC has its own backoff counter and they are independent one each other. Besides having $\mathrm{AC}$ parameter set of AIFSN [AC], $\mathrm{CW}_{\min }[\mathrm{AC}]$ and $\mathrm{CW}_{\max }[\mathrm{AC}]$, each $\mathrm{AC}$ in $\mathrm{AFR}+\mathrm{Q}$ scheme also has aggregate frame size parameter $\left(\mathrm{L}_{\mathrm{f}}\right)$ and fragment threshold size $\left(\mathrm{L}_{\text {frag }}\right)$.

Here we present the mechanism of transmitting the packet on AFR+Q scheme. The packet received by MAC layer will be stored in $\mathrm{AC}$ according to the priority value contained on each packet. Each AC with packet to be transmitted will perform the backoff procedure as in EDCA scheme. If the backoff counter has reached zero at the same time, AC with the highest priority will be selected to transmit the packet in advance, while the other AC will continue the backoff process by doubling the contention window size. Before transmitted, the packet size will be checked and if it exceeds the fragment threshold size $\left(\mathrm{L}_{\text {frag }}\right)$, the packet will be split up into fragment. Afterwards, the fragment will be added a FCS and is aggregated into a frame with an appropriate size of the parameter $\left(\mathrm{L}_{\mathrm{f}}\right)$. The large aggregate frame will then be transmitted. On the receiver side, the part of FCS on each fragment will be checked to determine an error of the fragment. 


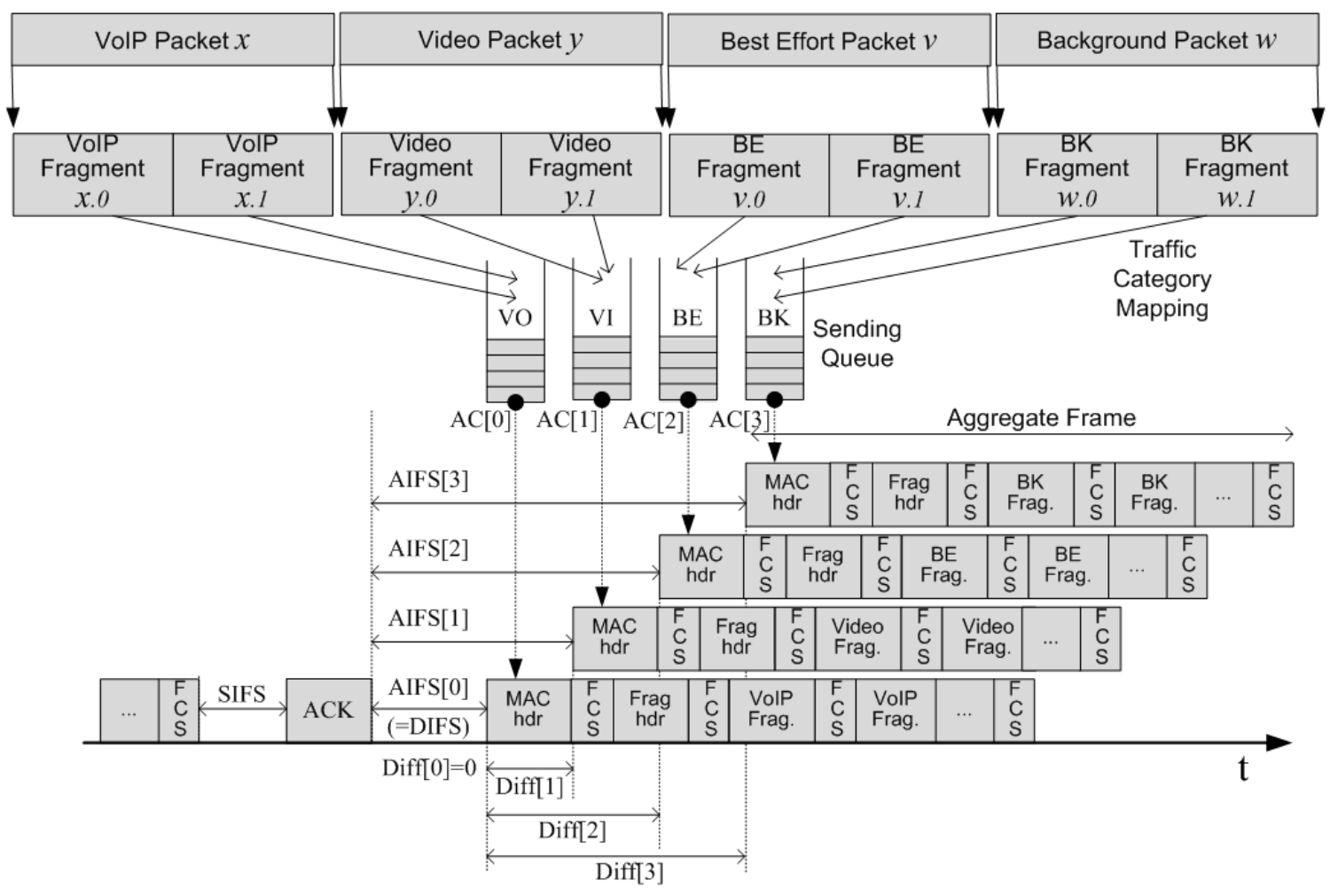

Fig. 1. AFR+Q Scheme

Similar to AFR scheme, if a fragment contains error, it will be sent back instead of the whole aggregate frame. In $A F R+Q$ scheme, we also specify two operational modes for transmitting aggregate frame, i.e., Basic Access and RTS/CTS access mode.

\section{RELATED WORK}

There are many studies have been conducted to propose a scheme for improving efficiency and throughput of the MAC layer as well as evaluating the performance given by each of these schemes. The efficiency of MAC layer is determined by measuring how much overhead is used. The overhead consists of backoff process, DIFS, MAC header, ACK, SIFS and PHY header. Some research have been propose different schemes to increase the throughput efficiency of MAC layer; among which are Burst ACK (Wu et al., 2013), Block ACK (Arif and Sari, 2012), Concatenation Packet (Hong and Tsai, 2010), Aggregation (Charfi et al., 2012) and AFR (Li et al., 2009).

From the literature survey, we conclude the research on the aggregation and fragmentation scheme effect on the performance of QoS scheme in IEEE 802.11 is still absent. However, it is worth noting that some studies have been conducted to analyze QoS scheme performance in 802.11 without considering the aggregation and fragmentation scheme.

QoS scheme performance analysis in IEEE 802.11, particularly EDCA scheme, can be seen in several papers. Most of the papers applied Markov chain approach to develop analytical model in analyzing EDCA scheme performance. The use of the Markov chain approach for DCF performance analysis was pioneered by (Bianchi, 2000). Bianchi (2000) model then improved by (Tinnirello and Bianchi 2010) which consider anomalous slot phenomenon in DCF scheme. Tinnirello and Bianchi (2010) model is later developed by (Han et al., 2012; Arif and Sari, 2012) so that it can be used to analyze the performance of EDCA scheme in IEEE 802.11p and IEEE 802.11n.

There are many other models proposed by researchers with respect to the service differentiation issue on EDCA using CW size and AIFS with different approach. Huang and Liao (2007) proposed average collision probability analysis for different contention 
zone during the AIFS period. Xu et al. (2009) proposed a detailed analytical model to evaluate the influence of all EDCA differentiation parameters to access delay (Xu et al., 2009). Inan et al. (2009) and Gas et al. (2011) proposed a three dimensional Markov chain to handle the differentiation effect of different $\mathrm{CW}$ size and AIFS duration on each AC accurately. Proposed a virtual collision analytical model between different access categories in one STA (Hu et al., 2008).

The literature survey shows that the analytical model for contention based of QoS with considering $\mathrm{CW}$, AIFS and virtual collision as well as considering the aggregation and packet fragmentation effect has not been conducted by any other research. In this study, we proposed an analytical model to evaluate AFR+Q scheme. Based on the proposed model, we demonstrated the saturation throughput achieved by each AC using a different CW size, AIFS duration, fragment size and frame size.

\section{ANALYTICAL MODEL}

This section presents the analytical model of $\mathrm{AFR}+\mathrm{Q}$ scheme in order to determine the saturation throughput achieved by AFR+Q scheme. We use the assumption that the queue of each AC is always has packets to be transmitted. Besides, to provide an accurate analytical model of AFR+Q scheme, we used assumption the channel condition is in error-prone.

\subsection{AFR+Q Markov Chain Model}

We use the bi-dimensional Markov chain for the analysis of saturation throughput of $A F R+Q$ scheme. Our proposed Markov chain model for AFR+Q scheme is shown in Fig. 2. AFR+Q has four queues to accommodate different traffic flows; the voice traffic in $\mathrm{AC}$ [0], the video traffic in $\mathrm{AC}$ [1], the best effort traffic in $\mathrm{AC}$ [2] and the background traffic in $\mathrm{AC}$ [3]. In the analysis, we use $\mathrm{AC}[\mathrm{z}]$ notation for each queue where $\mathrm{z}$ $\in\{0,1,2,3\}$. Each backoff counter state AC [z] of $\mathrm{AFR}+\mathrm{Q}$ is the bidimensional Markov process which represented by $\{\mathrm{s}(\mathrm{t}), \mathrm{b}(\mathrm{t})\}$, where $\mathrm{b}(\mathrm{t})$ is the stochastic process which represents the backoff counter condition $\mathrm{AC}[\mathrm{z}]$ in a range of 0 (the lowest counter position) up to $\mathrm{W}_{\mathrm{i}^{-1}}$ (the highest counter position) where $\mathrm{W}_{\mathrm{i}}$ is the minimum contention window size on the ${ }^{i}$ backoff counter stage. $\mathrm{s}(\mathrm{t})$ is the stochastic process which represents the backoff stage $\mathrm{AC}[\mathrm{z}]$ where on this $\mathrm{AFR}+\mathrm{Q}$ model the backoff stage of each $\mathrm{AC}[\mathrm{z}]$ is started from 0 (the lowest counter position) up to the $\mathrm{m}+\mathrm{f}$ stage, where $\mathrm{m}$ is the backoff stage limitation of the contention window size which can be doubled and $\mathrm{f}$ is the limitation of the aggregate frame retransmission attempt stage.

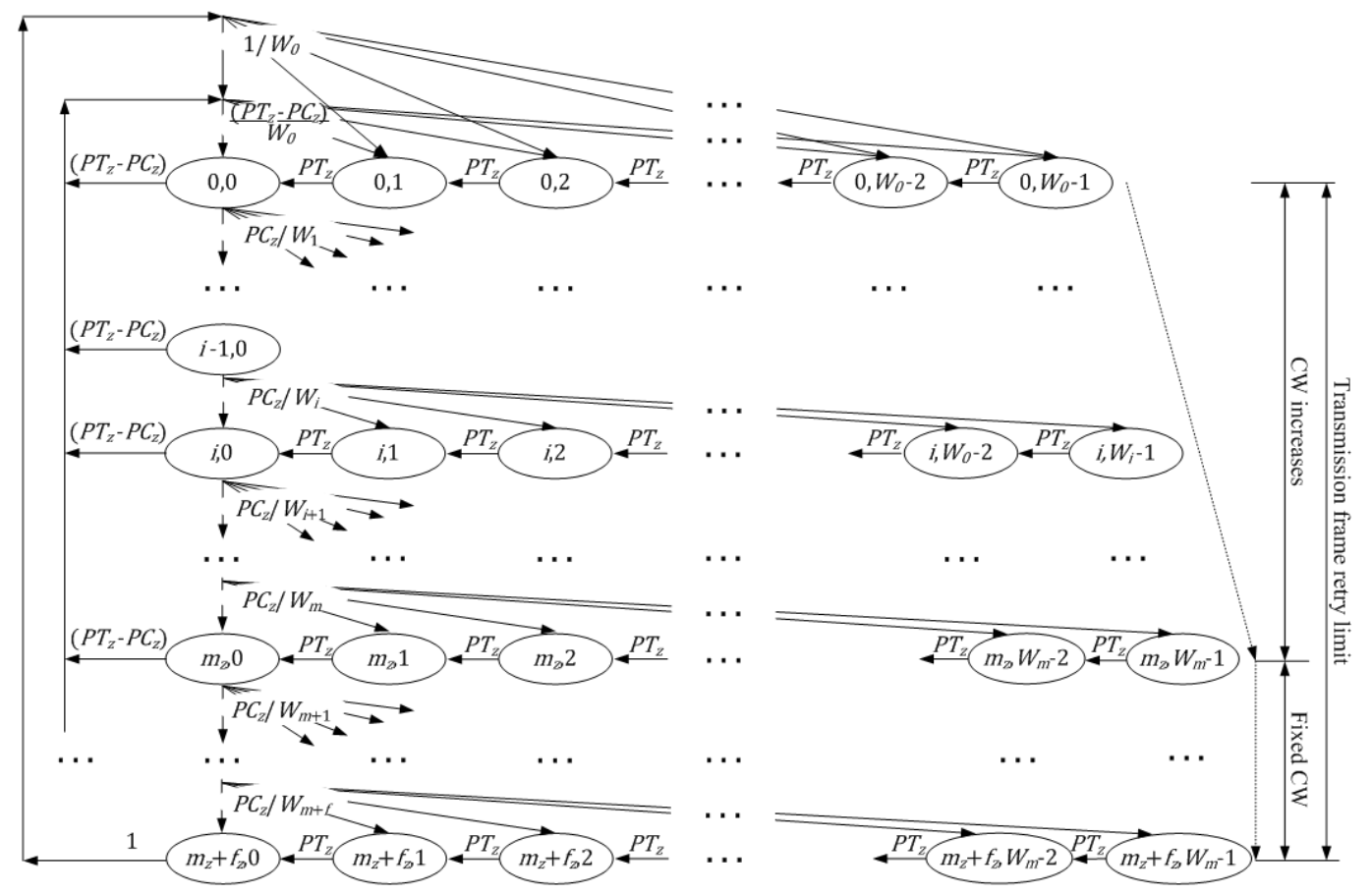

Fig. 2. Markov Chain model for AFR+Q 
The transition from one stage to a higher stage, e.g., from stage $\mathrm{i}-1$ to stage $\mathrm{i}$ will occur if $\mathrm{AC}[\mathrm{z}]$ fails to perform the transmission which is caused by either the busy state of a channel or the occurrence of a collision by $\mathrm{PC}_{\mathrm{z}}$. If it reaches the stage $m+f$ and the aggregate frame fails to be transmitted, then the aggregate frame will be removed from the queue and the backoff state will be transitioned back to the lowest stage to restart the other aggregate frame transmission. The transition from stage $0<\mathrm{i}<\mathrm{m}+\mathrm{f}$ to 0 stage will occur if $\mathrm{AC}[\mathrm{z}]$ has successfully performed the aggregate frame transmission.

In $\mathrm{AFR}+\mathrm{Q}$ mathematical model, $\mathrm{W}$ is minimum contention window size $\left(\mathrm{CW}_{\min }\right)$ and $\mathrm{m}$ is maximum backoff level. Therefore, $\mathrm{CW}_{\max }=2^{\mathrm{m}} \mathrm{W}$ and $\mathrm{W}_{\mathrm{i}}=2^{\mathrm{i}} \mathrm{W}$ where $i \in(0, \mathrm{~m})$ which called as the backoff stage. On the backoff stage $i \in(m+1, f)$, the Contention Window size can no longer be doubled. If the transmission can be successfully performed by each $\mathrm{AC}[\mathrm{z}]$ in all state $(\mathrm{i}, 0)$ where $0 \leq \mathrm{i}<\mathrm{m}+\mathrm{f}$, then the random backoff counter value will be selected between 0 to $\mathrm{W}_{\mathrm{o}}-1$. If the collision occurs in the state $(\mathrm{i}-1,0)$ for instance, then the random backoff counter value will be selected in a range of $(0$, $\left.\mathrm{W}_{\mathrm{i}}-1\right)$. The transition probability of Markov chain in AFR+Q can be formulated as follow Equation 1:

$$
\left\{\begin{array}{l}
p(i, k \mid i, k+1)=P T_{z}, k \in\left(1, W_{i}-1\right), \\
i \in\left(0, m_{z}+f_{z}\right) \\
p(0, k \mid i, 0)=\frac{\left(P T_{z}-P C_{z}\right)}{W_{0}}, k \in\left(0, W_{0}-1\right) \\
i \in\left(0, m_{z}+f_{z}-1\right) \\
p(i, k \mid i-1,0)=P C_{z} / W_{i}, k \in\left(0, W_{i}-1\right), \\
i \in\left(0, m_{z}+f_{z}\right) \\
p\left(0, k \mid m_{z}+f_{z} 0\right)=1 / W_{0}, k \in\left(0, W_{o}-1\right)
\end{array}\right.
$$

$\mathrm{m}_{\mathrm{z}}$ is the maximum number of contention window can be doubled, $\mathrm{m}_{\mathrm{z}}+\mathrm{f}_{\mathrm{z}}$ is retransmission limit, $\mathrm{W}_{0}$ is the minimum contention window size, $\mathrm{W}_{\mathrm{i}}$ is the window size at stage $\mathrm{i}\left(\mathrm{e} . \mathrm{g} ., \mathrm{W}_{\mathrm{i}}=\mathrm{W}_{0} \times 2^{\mathrm{i}}\right.$ for $\mathrm{i} \in(0, \mathrm{M}-1)$ and $\mathrm{W}_{\mathrm{i}}=\mathrm{W}_{\mathrm{m}}$ for $i \in\left(\mathrm{m}_{\mathrm{z}}, \mathrm{m}_{\mathrm{z}}+\mathrm{f}_{\mathrm{z}}\right), \mathrm{PT}_{\mathrm{z}}$ is the probability of backoff counter can be decremented in a time slot and $\mathrm{PC}_{\mathrm{z}}$ is the collision probability in each transmission attempt.

\subsection{AFR+Q Transmission and Collision Probability}

In AFR+Q Markov chain model, it is assumed that in a transmission attempt, the aggregate frame will possibly experience collision with probability equal to
$P C_{z}$. In other words, $\mathrm{PC}_{\mathrm{z}}$ is the probability where there is only one $\mathrm{AC}[\mathrm{z}]$ from $4 \mathrm{n}-1$ AC [z] can perform transmission successfully in one time slot. Each $\mathrm{AC}[\mathrm{z}]$ can be collide with other AC [z] in the same STA (virtual collision, denoted as $\mathrm{Pl}_{\mathrm{z}}$ ). Collision can also happen with other STA (external collision, denoted as $\mathrm{PO})$. Therefore, the collision probability on each $\mathrm{AC}[\mathrm{z}]$ can be expressed as Equation 2:

$\mathrm{PC}_{\mathrm{z}}=\mathrm{PI}_{\mathrm{z}}+\left(1-\mathrm{PI}_{\mathrm{z}}\right) \mathrm{P} 0$

Each AC [z] experienced virtual collision only with $\mathrm{AC}[\mathrm{z}]$ in higher priority in the same STA, therefore the virtual collision probability of $\mathrm{AC}[0]$ is $\mathrm{PI}_{0}=0$. The collision probability $\mathrm{PC}_{0}=\mathrm{PI}_{0}+\left(1-\mathrm{pI}_{0}\right) \mathrm{P} 0=\mathrm{P} 0$ or in other words the external collision probability on each $\mathrm{AC}$ [z] where $0<\mathrm{z} \leq 3$ is equal to $\mathrm{PC}_{0}$.

Stationary distribution of the Markov chain can be expressed as $b_{i, k}=\lim _{t \rightarrow \infty} P\{s(t)=i, b(t)=k\}, i \in(0, m+f)$, $\mathrm{k} \in\left(0, \mathrm{CW}_{\mathrm{i}}-1\right)$. The transmission occurs when the counter backoff is equal to zero. That means the transmission of a AC $[z]$ denoted by $\tau_{z}$ is the sum of the state backoff $b_{i, 0}$ and can be expressed as Equation 3:

$$
\tau_{\mathrm{z}}=\sum_{\mathrm{i}=0}^{\mathrm{m}+\mathrm{f}-1} \mathrm{~b}_{\mathrm{i}, 0}
$$

According to the Markov chain model for AFR+Q in Fig. 2, the stationary distribution of the chain for $b_{i, 0}$, $b_{m+f-1,0}, b_{m+f, 0}$ and $b_{i, k}$ are as follow Equation 4:

$$
\left\{\begin{array}{l}
b_{i, 0}=P C_{z}^{i} b_{0,0}, 0<i<m+f-2 \\
b_{m+f-1,0}=\frac{P_{z}^{m+f-1}}{P_{z}-P C_{z}} b_{0,0} \\
b_{m+f, 0}=P_{z}^{m+f} b 0,0 \\
b_{i, k}=\frac{W_{i}-k}{W_{i}} b_{i, 0}, i \in(0, m), k \in\left(0, W_{i}-1\right)
\end{array}\right.
$$

The sum of all $b_{i, k}$ state in Markov model is equal to one, then Equation 5:

$$
\begin{aligned}
& 1=\sum_{i=0}^{m_{Z}+f_{Z}} \sum_{k=0}^{w_{i}-1} b_{i, k}=\sum_{i=0}^{m_{Z}+f_{Z}} b_{i, 0} \frac{W_{i}+1}{2} \\
& =\frac{\mathrm{b}_{0,0}}{2}\left[\begin{array}{l}
\sum_{\mathrm{i}=0}^{\mathrm{m}_{\mathrm{Z}}-1}\left(\left(2 \mathrm{PC}_{\mathrm{Z}}\right)^{\mathrm{i}} \mathrm{W}_{\mathrm{Z}}+\mathrm{PC}_{\mathrm{Z}}^{\mathrm{i}}\right)+\left(2^{\mathrm{m}_{\mathrm{Z}}} \mathrm{W}_{\mathrm{Z}}+1\right) \\
\times\left(\sum_{\mathrm{i}=\mathrm{m}_{\mathrm{Z}}}^{\mathrm{m}_{\mathrm{Z}}+\mathrm{f}^{-2}} \mathrm{PC}_{\mathrm{z}}^{\mathrm{i}}+\frac{\mathrm{PC}_{\mathrm{Z}}^{\mathrm{m}_{\mathrm{Z}}+\mathrm{f}^{-1}}}{\mathrm{PT}_{\mathrm{Z}}-\mathrm{PC}_{\mathrm{Z}}}+\mathrm{PC}_{\mathrm{Z}}^{\mathrm{m}_{\mathrm{Z}}+\mathrm{f}_{\mathrm{Z}}}\right)
\end{array}\right]
\end{aligned}
$$


Based on Equation (5), we can derive the probability of $b_{0,0}$ state as shown in Equation (6) and the transmission probability of $\mathrm{AC}[\mathrm{z}]$ as shown in Equation (7):

$$
\begin{aligned}
& \mathrm{b}_{0,0}=\frac{2}{\sum_{\mathrm{i}=0}^{\mathrm{m}_{\mathrm{z}}^{-1}}\left(\left(2 \mathrm{PC}_{\mathrm{z}}\right)^{\mathrm{i}} \mathrm{W}_{\mathrm{z}}+\mathrm{PC}_{\mathrm{z}}^{\mathrm{i}}\right)+\left(2^{\mathrm{m}_{\mathrm{z}}} \mathrm{W}_{\mathrm{z}}+1\right)} \\
& \left(\sum_{\mathrm{i}=\mathrm{m}_{\mathrm{z}}}^{\mathrm{m}_{\mathrm{z}}+\mathrm{f}_{\mathrm{z}}-2} \mathrm{PC}_{\mathrm{z}}^{\mathrm{i}}+\frac{\mathrm{PC}_{\mathrm{z}}^{\mathrm{m}_{2}+\mathrm{f}_{\mathrm{z}}-1}}{\mathrm{PT}_{\mathrm{z}}-\mathrm{PC}_{\mathrm{z}}}+\mathrm{PC}_{\mathrm{z}}^{\mathrm{m}_{\mathrm{z}}+\mathrm{f}_{\mathrm{z}}}\right) \\
& \tau_{\mathrm{z}}=\sum_{\mathrm{i}=0}^{\mathrm{m}_{\mathrm{z}}+\mathrm{fz}} \mathrm{b}_{\mathrm{i}, 0}=\mathrm{b}_{0,0} \sum_{\mathrm{i}=0}^{\mathrm{m}_{\mathrm{z}}+\mathrm{fz}} \mathrm{PC}_{\mathrm{z}}^{\mathrm{i}} \\
& =\frac{2 \sum_{\mathrm{i}=\mathrm{o}}^{\mathrm{m}_{\mathrm{z}}+\mathrm{fz}} \mathrm{PC}_{\mathrm{z}}^{\mathrm{i}}}{\sum_{\mathrm{i}=0}^{\mathrm{m}_{\mathrm{z}}^{-1}}\left(\left(2 \mathrm{PC}_{\mathrm{z}}\right)^{\mathrm{i}} \mathrm{W}_{\mathrm{z}}+\mathrm{PC}_{\mathrm{z}}^{\mathrm{i}}\right)+\left(2^{\mathrm{m}_{\mathrm{z}}} \mathrm{W}_{\mathrm{z}}+1\right)} \\
& \left(\sum_{\mathrm{i}=\mathrm{m}_{\mathrm{z}}}^{\mathrm{m}_{\mathrm{z}}+\mathrm{f}_{\mathrm{z}}-2} \mathrm{PC}_{\mathrm{z}}^{\mathrm{i}}+\frac{\mathrm{PC}_{\mathrm{z}}^{\mathrm{m}_{2}+\mathrm{f}_{\mathrm{z}}-1}}{\mathrm{PT}_{\mathrm{z}}-\mathrm{PC}_{\mathrm{z}}}+\mathrm{PC}_{\mathrm{z}}^{\mathrm{m}_{\mathrm{z}}+\mathrm{f}_{\mathrm{z}}}\right)
\end{aligned}
$$

Transmission probability a STA that is affected by the virtual collision Equation 8:

$$
\sigma_{\text {total }}=\sum_{\mathrm{z}=0}^{3} \sigma_{\mathrm{z}}
$$

\subsection{AFR+Q Saturation Throughput}

The saturation throughput of AFR+Q scheme is determined by: (i) the probability that at least one STA transmits in a time slot (PTR), (ii) the probability that a transmission attempt of $\mathrm{AC}[\mathrm{z}]$ is successful given that there is at least one station transmitting in a time slot $\left(\mathrm{PS}_{\mathrm{z}}\right)$, (iii) the probability that a transmission attempt fails due to a collision given that there is at least one station transmitting in a time slot (PFC) and (iv) the probability that a time slot is in idle state. PTR, $\mathrm{PS}_{\mathrm{z}}$ and PFC can be expressed as follow Equation 9:

$$
\begin{aligned}
& \text { PTR }=1-\left(1-\sigma_{\text {total }}\right)^{\mathrm{n}} \\
& \mathrm{PS}_{\mathrm{z}}=\frac{\mathrm{n} \times \sigma_{\mathrm{z}} \times\left(1-\sigma_{\text {total }}\right)^{\mathrm{n}-1}}{\mathrm{PTR}}, \mathrm{z}=0,1,2,3 \\
& \mathrm{PFC}=\frac{1-\left(1-\sigma_{\text {total }}\right)^{\mathrm{n}}-\mathrm{n} \times \sigma_{\text {total }} \times\left(1-\sigma_{\text {total }}\right)^{\mathrm{n}-1}}{\operatorname{PTR}}
\end{aligned}
$$

Suppose $S_{z}$ is the saturation throughput of AC [z], then the saturation throughput of $\mathrm{AC}$ [z] can be calculated using Equation (10). In Equation (10), $\mathrm{E}\left[\mathrm{L}_{\mathrm{z}}\right]$ is the average aggregate frame size of $\mathrm{AC}[\mathrm{z}], \mathrm{t}_{\mathrm{sz}}$ is successful time to transmit an aggregate frame while $t_{\mathrm{cz}}$ is collision time.
The aggregate frame size in $A F R+Q$ scheme is given by the frame size $\left(\mathrm{L}_{\mathrm{f}}\right)$ and fragment error rate $\left(\mathrm{P}_{\mathrm{e}}^{\text {frag }}\right)$, which $\mathrm{E}[\mathrm{L}]=\mathrm{L}_{\mathrm{f}}\left(1-\mathrm{P}_{\mathrm{e}}{ }^{\text {frag }}\right)$ and $\mathrm{P}_{\mathrm{e}}^{\text {frag }}=1-(1-$ $\left.\mathrm{P}_{\mathrm{b}}\right)^{\text {Lfrag }+ \text { LFcs }}$. With substitution $\mathrm{E}[\mathrm{L}]$ and $\mathrm{P}_{\mathrm{e}}^{\text {frag }}$ to Equation (10), we can derive the equation for saturation throughput of each AC [z] in AFR+Q scheme as in (11), where $p_{b}$ is the BER of wireless channel, $L_{f}$ is the aggregate frame size, $\mathrm{L}_{\text {frag }}$ is the fragment size and $\mathrm{L}_{\mathrm{Fcs}}$ is the length of FCS.

\section{SIMULATION AND RESULTS}

We use Matlab to simulate our AFR+Q analytical model. As explained before, the throughput of $\mathrm{AC}[\mathrm{z}]$ transmitting aggregate frames is determined by how much the transmission probability $\left(\tau_{\mathrm{z}}\right)$ achieved by a $\mathrm{AC}[\mathrm{z}]$. Based on the Equation (7), the transmission probability on each $\mathrm{AC}[\mathrm{z}]$ is affected by the collision probability $\left(\mathrm{PC}_{\mathrm{z}}\right)$, the maximum level of backoff stage $\left(\mathrm{m}_{\mathrm{z}}\right)$, the attempt limitation for retransmitting aggregate frame $\left(f_{z}\right)$, the minimum contention window size $\left(\mathrm{W}_{\mathrm{z}}\right)$ and the probability of $\mathrm{AC}[\mathrm{z}]$ can decrement its backoff counter $\mathrm{PT}_{\mathrm{z}}$.

In order to find out the saturation throughput of AFR+Q scheme, we use the parameters values as shown in Table 1. The data rate to represent very high speed WLAN IEEE $802.11 \mathrm{n}$ condition that we apply is 120 Mbps. In simulation we used RTS/CTS access mode, which the RTS frame length is 20 bytes and the CTS frame length is 14 bytes.

Figure 3 shows the saturation throughput for each $\mathrm{AC}[\mathrm{z}]$. The saturation throughput of AC [z] increases when there is only a small number of STA and decreases as the number of STA becomes higher. The decreasing of $\mathrm{AC}[\mathrm{z}]$ saturation throughput is caused by the decreasing of successful transmission probability and the increasing of collision probability while the number of STA is increase. When the number of STA is 2, $\mathrm{AC}$ [0] throughput becomes lower than $\mathrm{AC}$ [1] throughput in which is caused by using smaller AC [0] aggregate frame size compared to the aggregate frame used by $\mathrm{AC}[1]$. On the other hand, throughput of $\mathrm{AC}$ [2] becomes higher than AC [3] when their aggregate frame size is the same. This is due to the size of the minimum contention window and AIFS period of AC [2] is smaller than the AC [3]. As the result of using a smaller contention window size of a $\mathrm{AC}[\mathrm{z}]$, the transmission probability will be bigger than $\mathrm{AC}[\mathrm{z}]$ which has bigger contention window. 
Teuku Yuliar Arif and Riri Fitri Sari / Journal of Computer Science 9 (10): 1356-1368, 2013

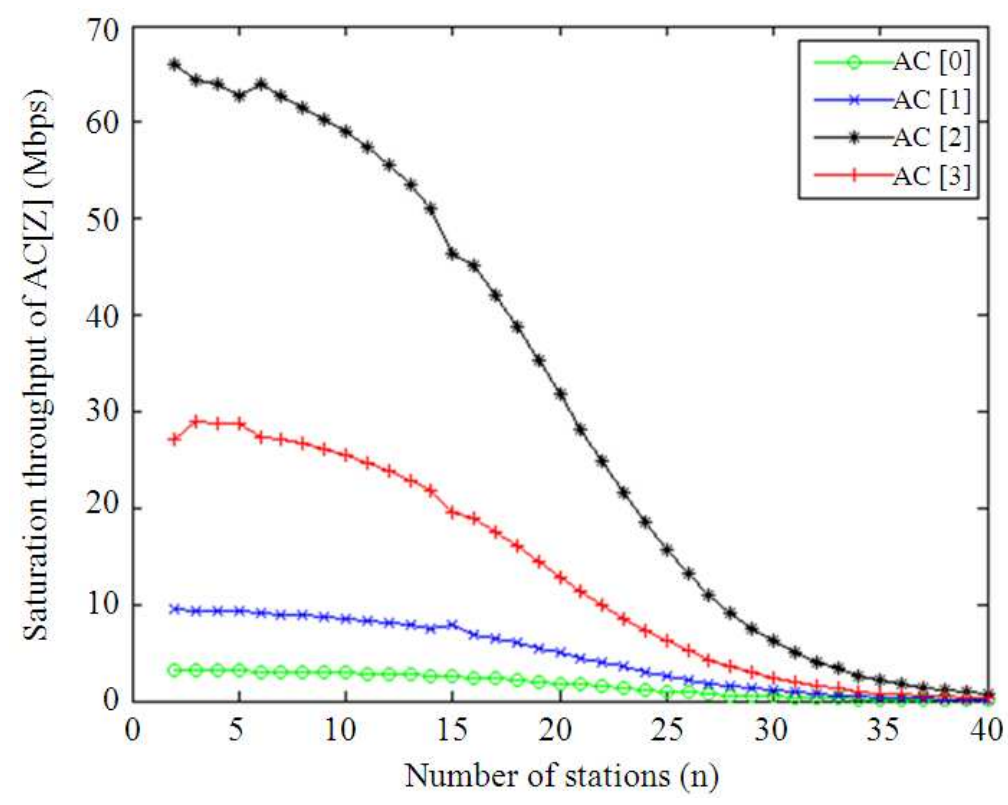

Fig. 3. The throughput of each $\mathrm{AC}[\mathrm{z}]$

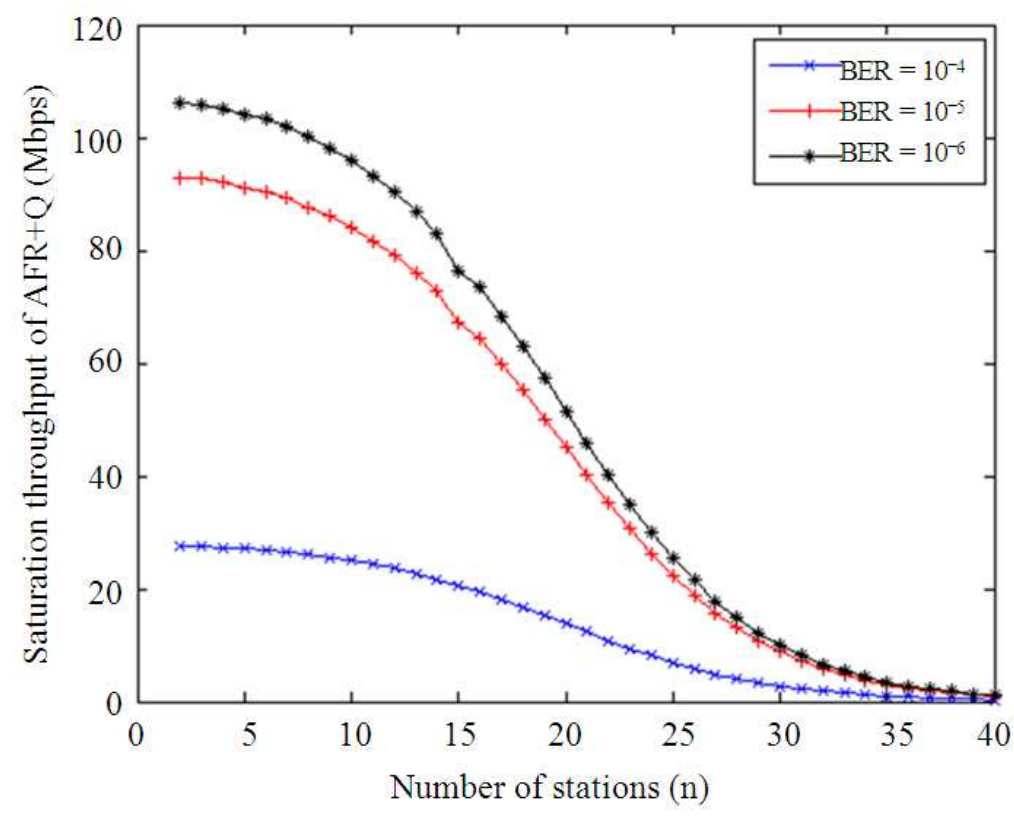

Fig. 4. Saturation Throughput Vs. Number of station

$$
\begin{aligned}
& \mathrm{S}_{\mathrm{z}}= \frac{\mathrm{PS}_{\mathrm{z}} \times \mathrm{PTR} \times \mathrm{E}\left[\mathrm{L}_{\mathrm{z}}\right]}{(1-\mathrm{PTR}) \times \text { aTimeSlot }+} \\
& \sum_{\mathrm{Z}=0}^{3} \mathrm{PTR} \times \mathrm{PS}_{\mathrm{Z}} \times \mathrm{t}_{\mathrm{S}_{\mathrm{z}}}+\mathrm{PTR} \times \mathrm{PFC} \times \mathrm{t}_{\mathrm{C}_{\mathrm{z}}}
\end{aligned}
$$

$$
\begin{aligned}
\mathrm{S}_{\mathrm{zAFR}+\mathrm{Q}}= & \frac{\mathrm{PS}_{\mathrm{i}} \times \mathrm{PTR} \times \mathrm{L}_{\mathrm{f}_{\mathrm{Z}}}\left(\left(1-\mathrm{p}_{\mathrm{b}}\right)^{\mathrm{L}_{\text {frag }}+\mathrm{L}_{\mathrm{FCS}}}\right)}{(1-\mathrm{PTR}) \times \mathrm{aTimeSlot}+} \\
& \sum_{\mathrm{Z}=0}^{3} \mathrm{PTR} \times \mathrm{PS}_{\mathrm{Z}} \times \mathrm{t}_{\mathrm{S}_{\mathrm{Z}}}+\mathrm{PTR} \times \mathrm{PFC} \times \mathrm{t}_{\mathrm{C}}
\end{aligned}
$$


Table 1. The simulation parameters for AFR+Q

\begin{tabular}{ll}
\hline Parameter & Value \\
\hline Data transmission rate & $120 \mathrm{Mbps}$ \\
Control message trans. Rate & $120 \mathrm{Mbps}$ \\
An idle slot time & $9 \mu \mathrm{sec}$ \\
SIFS time & $16 \mu \mathrm{sec}$ \\
DIFS = SIFS+2slot & $34 \mu \mathrm{sec}$ \\
Propagation delay & $1 \mu \mathrm{sec}$ \\
Maximum station number & 40 \\
RTS frame length & 20 bytes \\
CTS frame length & 14 bytes \\
PHY layer overhead & 20 bytes \\
MAC sub layer overhead & 38 bytes \\
ACK frame length & 46 bytes \\
FSC length & 2 bytes \\
BER & 10 -6 \\
AC[z]retry limit & {$[3,7,7,7]$} \\
AC[0]contention window min. & 15 \\
AC[0]fragment size & 128 bytes \\
AC[0]aggregate frame length & 1024 bytes \\
AIF[0] & SIFS+2slots \\
AC[1] contention window min. & 31 \\
AC[1]fragment size & 512 bytes \\
AC[1] aggregate frame length & 8192 bytes \\
AIFS[1] & SIFC +3 slots \\
AC[2] contention window min. & 31 \\
AC[2] fragment size & 2048 bytes \\
AC[2] ] aggregate frame length & 65536 bytes \\
AIFS[2] & SIFS +4 slots \\
AC[3] ] contention window min. & 63 \\
AC[3] fragment size & 2048 bytes \\
AC[3] aggregate frame length & 65536 bytes \\
AIFS[3] & SIFS +5 slots \\
\hline
\end{tabular}

Based on the Equation (11), the saturation throughput on each AC $[\mathrm{z}]$ in $\mathrm{AFR}+\mathrm{Q}$ STA is determined by the aggregate frame size, the fragment size and wireless BER channel. The aggregate throughput of $\mathrm{AFR}+\mathrm{Q}$ is the sum of each AC [z] throughput saturation and can be expressed as $\mathrm{S}_{\text {total }}=\sum_{\mathrm{z}=0}^{3} \mathrm{~S}_{\mathrm{ZAFR}+\mathrm{Q}}$. By using the same parameter as shown in Table 1, the saturation throughput characteristic of $\mathrm{AC}[\mathrm{z}]$ in different BER is shown in Fig. 4. When the BER channel is $10^{-6}$ and the number of STA is equal to 2, the aggregate throughput of AFR+Q scheme would be around $106 \mathrm{Mbps}$. This indicates that AFR+Q scheme has higher efficiency on MAC layer since the aggregate throughput is slightly below the data rate used, which is $120 \mathrm{Mbps}$.
Table 2. Parameters used for experiment in Fig. 7 and 8

\begin{tabular}{ll}
\hline Parameter & Value \\
\hline Data transmission rate & $120 \mathrm{Mbps}$ \\
Control message trans. Rate & $120 \mathrm{Mbps}$ \\
An idle slot time & $9 \mu \mathrm{sec}$ \\
SIFS time & $16 \mu \mathrm{sec}$ \\
DIFS = SIFS+2slot & $34 \mu \mathrm{sec}$ \\
Propagation delay & $1 \mu \mathrm{sec}$ \\
Station number & 2 \\
RTS frame length & 20 bytes \\
CTS frame length & 14 bytes \\
PHY layer overhead & $20 \mu \mathrm{sec}$ \\
AFR+Q MAC sublayer overhead & 38 bytes \\
EDCA MAC sublayer overhead & 28 bytes \\
AFR+Q ACK frame length & 6 bytes \\
EDCA ACK frame length & 14 bytes \\
AFR+Q FCS length & 2 bytes \\
BER & $10^{-5}$ \\
AFR+Q aggregate payload length & 8192 bytes \\
AFR+Qfragmentthreshold & 128 bytes \\
AC[0] contention window min. & 15 \\
AIFS[0] & SIFS +2 slots \\
AC[1] contention window min. & 31 \\
AIFS[1] & SIFS +3 slots \\
AC[2] contention window min. & 31 \\
AIFS[2] & SIFS +4 slots \\
AC[3] contention window min. & 63 \\
AIFS[3] & SIFS +5 slots \\
\hline
\end{tabular}

The effect of fragment size on the saturation throughput of AFR $+\mathrm{Q}$ scheme is shown in Fig. 5. Simulation result shows the increased fragmention size caused the throughput of AFR+Q scheme decreases. This is due to the size of fragment as exponential function of BER condition, or in other words, the larger size of fragment makes error probability of the fragment become higher and causes the decreasing throughput. The same thing also happens when the fragment size remains constant but the channel condition of BER becomes higher which resulting higher error probability of the fragment and leads to a decreased AFR+Q throughput.

The effect of aggregate frame on the saturation throughput of AFR+Q scheme is shown in Fig. 6. Simulation result shows a larger aggregate frame used produces a higher throughput on AFR+Q scheme. According to the Equation (11), a larger value of Lfz will increase the throughput. If the BER condition gets higher, the larger aggregate frame will decrease the throughput. This condition happens when the aggregate frame size is large so the error probability of the frame will increase as well. 


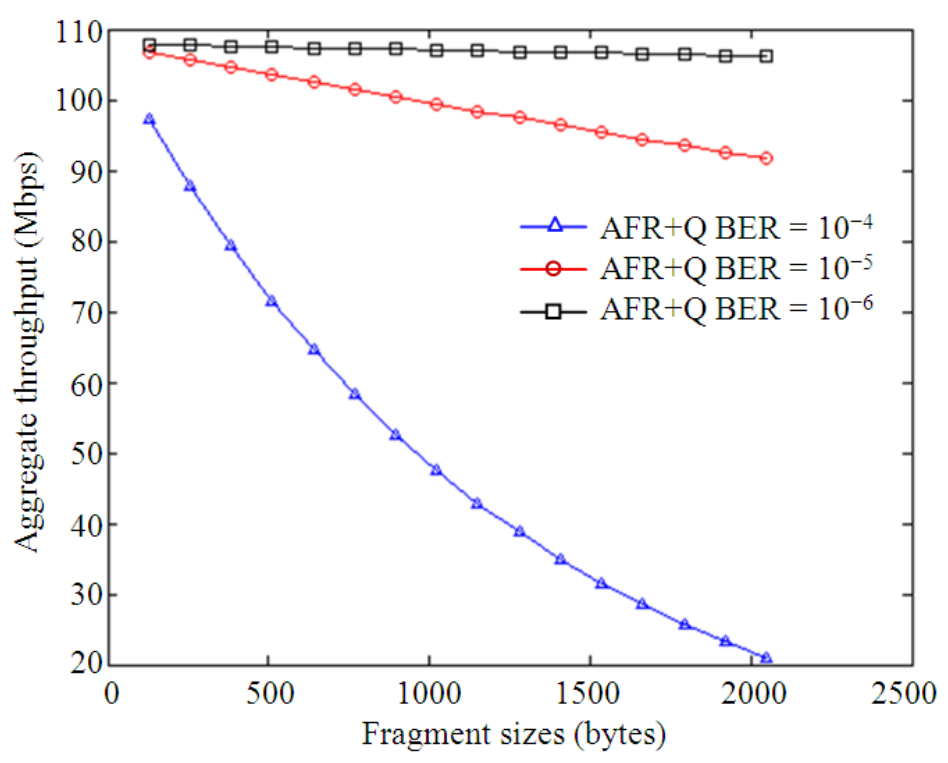

Fig. 5. Throughput Vs. increased fragment size

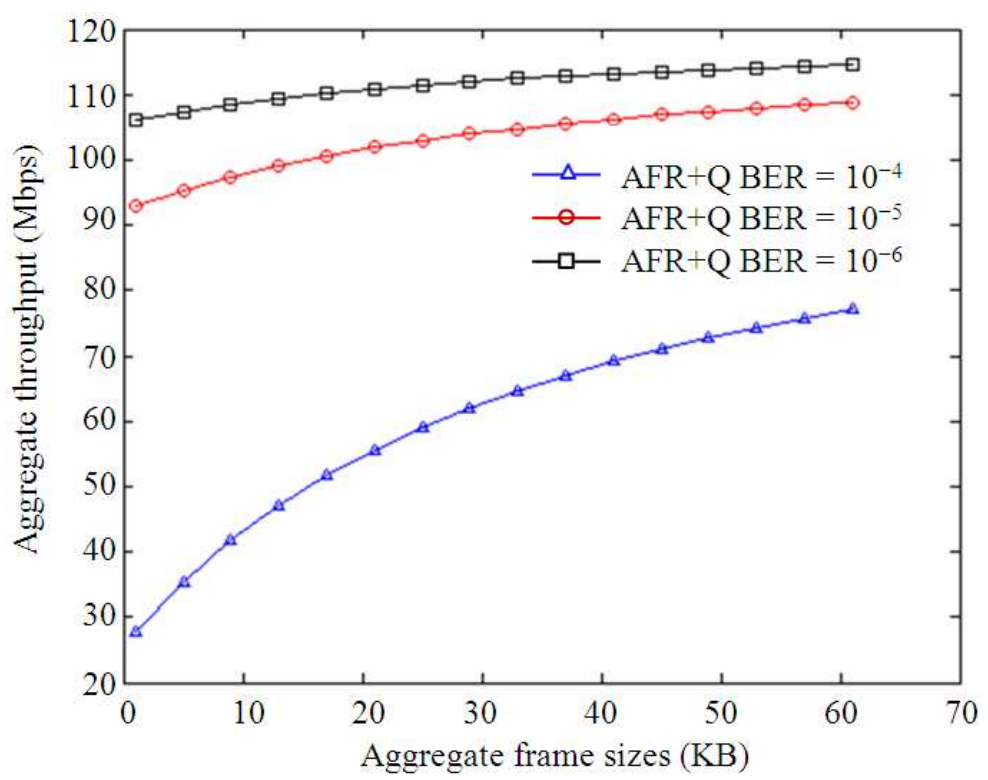

Fig. 6. Throughput Vs. increased frame size

The main difference between the AFR+Q and EDCA schemes is that AFR+Q scheme performs packet fragmentation and aggregation before transmission. FCS will be inserted between fragments and will be used by the receiver to check if the fragment is having an error. When error is found in the fragment, the receiver will only request erroneous fragment to be sent back. On the other hand, EDCA scheme does not apply such fragmentation mechanism and aggregate frame.

Table 2 shows the parameters that are used to investigate $\mathrm{AFR}+\mathrm{Q}$ scheme performance compared with EDCA scheme. 
Teuku Yuliar Arif and Riri Fitri Sari / Journal of Computer Science 9 (10): 1356-1368, 2013

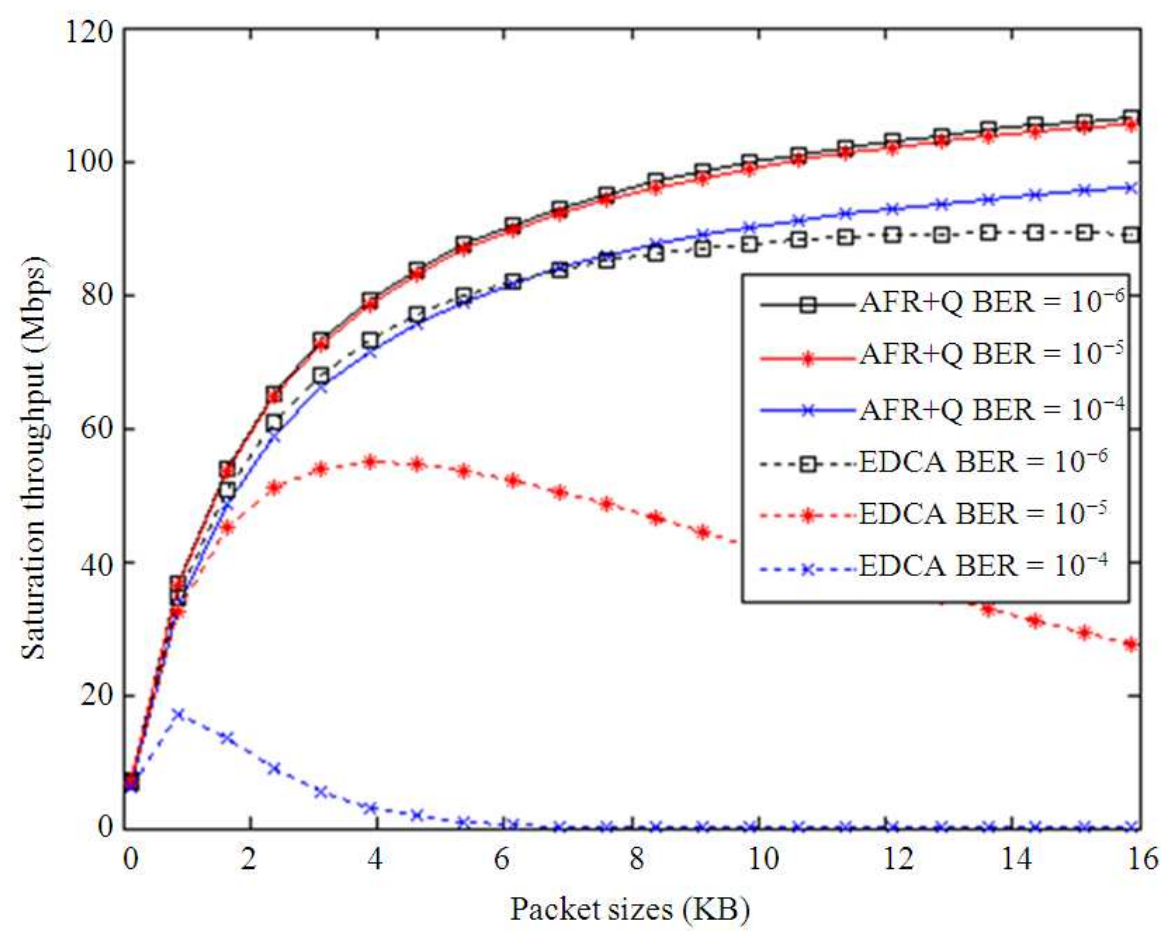

Fig. 7. AFR $+Q$ Vs. EDCA saturation throughput

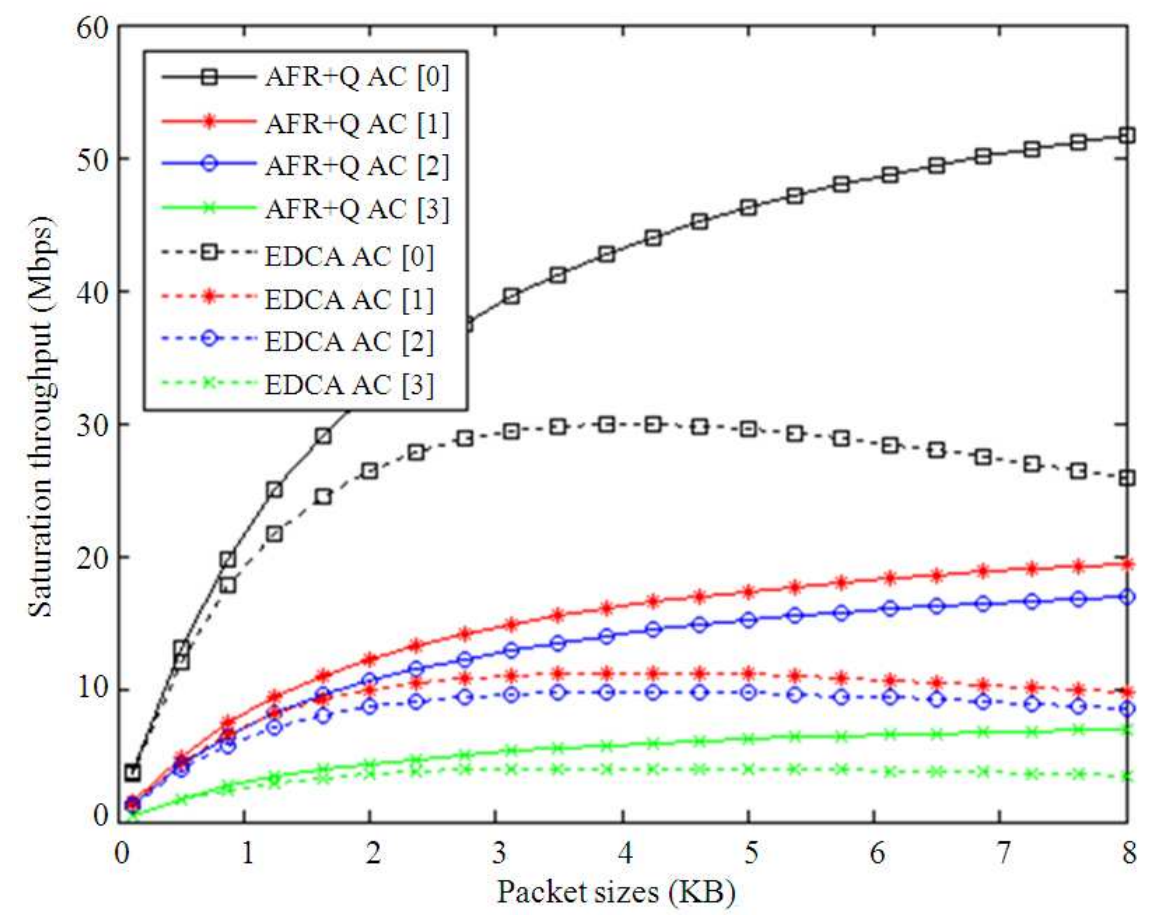

Fig. 8. Saturation throughput in different BER channel 
We use transmission probability assumption for AC [0] $=0.025$ and by using Equation (7), we get $\mathrm{AC}[1]=$ $0.0523, \mathrm{AC}[2]=0.0483$ and $\mathrm{AC}[3]=0.0209$. AFR+Q ACK frame length is 46 bytes and AFR+Q MAC sub layer overhead is 38 bytes. We also used the EDCA parameter value where EDCA ACK frame length is 14 bytes and EDCA MAC sub layer overhead is 28 bytes.

The comparison of saturation throughput of AFR+Q and EDCA schemes is shown in Fig. 7. The simulation result shows that the saturation throughput of each AC $[\mathrm{z}]$ increased if the transmitted packet size using AFR+Q is increased. AFR $+\mathrm{Q}$ scheme produces throughput on $\mathrm{AC}[\mathrm{z}]$ traffic higher than the throughput on $\mathrm{AC}[\mathrm{z}]$ in EDCA scheme. Overall, aggregate throughput achieved on MAC layer of AFR+Q is much better than the aggregate throughput achieved by EDCA scheme. Such higher throughput is achieved by using a larger frame size on AFR+Q compared to EDCA.

The usage of packet fragmentation and aggregate frame also cause the throughput of $A F R+Q$ is higher than throughput of EDCA scheme as shown in Fig. 8. The result of the simulation shows the aggregate throughput of AFR+Q scheme, in which the mechanism of fragmentation is used, will be helpful since the mechanism of fragmentation can reduce the error probability of the frame transmitted along the fragment size. It is contrary to the EDCA scheme, where error probability might occur along the frame size during the transmission. Therefore the aggregate throughput generated by AFR+Q is much better than EDCA scheme.

\section{CONCLUSION}

In this study we developed the AFR scheme by adding QoS capability to its original scheme and call it as AFR+Q scheme. We have also derived an analytical model of AFR+Q scheme and analyzed its performance. Simulation of the analytical model was conducted using Matlab. We observed that the performance of AFR+Q has a higher MAC layer saturation throughput compared to the EDCA scheme. The throughput of $\mathrm{AC}[\mathrm{z}]$ in AFR+Q scheme is higher compared to throughput of AC [z] in EDCA scheme. The higher throughput on AFR+Q scheme caused by it has the better transmission probability, using packet fragmentation and aggregate frame mechanism.

In the future works, we will apply AFR+Q scheme with next generation WLAN. The future WLAN based the new candidate standards of IEEE 802.11ac and 802.11ad. The PHY layer of both standards will use up to $160 \mathrm{MHz}$ bandwidth of channel, 256 QAM, 8 spatial streams and Multi user MIMO (MU-MIMO) to provide up to $6 \mathrm{Gbps}$ of data rate. The MAC layer of both standards is targeted to provide up to 1 Gbps throughput and be able to transmit MPDU simultaneously to four different user at the same time. To provide the compatibility with the previous standards, we will design the new PLCP header for both standards. We will also design the new MAC to provide the capability of MPDU transmission simultaneously using MU-MIMO.

\section{REFERENCES}

Arif, T.Y. and R.F. Sari, 2012. An analytical model of A-MSDU scheme with enhanced Block ACK for IEEE 802.11n networks. Proceedings of the 8th IEEE International Conference on Networks, Dec. 12-14, IEEE Xplore Press, Singapore, pp: 2910-298. DOI: 10.1109/ICON.2012.6506572

Bianchi, G., 2000. Performance analysis of the IEEE 802.11 distributed coordination function. IEEE J. Selected Areas Commun., 18: 535-547. DOI: 10.1109/49.840210

Charfi, E., L. Chaari and L. Kamoun, 2012. Analytical analysis of applying aggregation with fragment retransmission on IEEE 802.11e EDCA network in saturated conditions. Proceedibns of the Third International Conference on Communications and Networking, Mar. 29-Apr. 1, IEEE Xplore Press, Hammamet, pp: 1-9. DOI: 10.1109/ComNet.2012.6217730

Gas, M., K. Kosek-Szott, M. Natkaniec and A.R. Pach. 2011. 3D Markov chain-based saturation throughput model of IEEE 802.11 EDCA. Electron. Lett., 47: 826-827. DOI: 10.1049/el.2011.0693

Han, C., M. Dianati, R. Tafazolli, R. Kernchen and X. Shen, 2012. Analytical study of the IEEE 802.11p MAC sublayer in vehicular networks. IEEE Intell. Trans. Syst., 13: 873-886. DOI: 10.1109/TITS.2012.2183366

Hong, W. and Z. Tsai, 2010. A multichannel scheduler for high-speed wireless backhaul links with packet concatenation. IEEE Trans. Mob. Comput., 9: 201214. DOI: 10.1109/TMC.2009.110

Hu, J., G. Min, M.E. Woodward and W. Jia, 2008. A comprehensive analytical model for IEEE 802.11e QoS differentiation schemes under unsaturated traffic loads. Proceedings of the IEEE International Conference on Communications, May 19-23, IEEE Xplore Press, Beijing, pp: 241-245. DOI: 10.1109/ICC.2008.52 
Huang, C. and W. Liao, 2007. Throughput and delay performance of IEEE 802.11e enhanced distributed channel access (EDCA) under saturation condition. IEEE Trans. Wireless Commun., 6: 136-145. DOI: 10.1109/TWC.2007.04796

IEEE Std. 802.11, 1999. Part 11: Wireless LAN Medium Access Control (MAC) and Physical Layer (PHY) Specifications.

IEEE Std. 802.11e, 2005. Wireless LAN Medium Access Control (MAC) and Physical Layer (PHY) specifications Amendment 8: Medium Access Control (MAC) Quality of Service Enhancements.

IEEE Std. 802.11e, 2009. Part 11: Wireless LAN Medium Access Control (MAC)and Physical Layer (PHY) Specifications Amendment 5: Enhancements for Higher Throughput.

Inan, I., F. Keceli and E. Ayanoglu, 2009. Analysis of the 802.11e enhanced distributed channel access function. IEEE Trans. Commun., 57: 1753-1764. DOI: 10.1109/TCOMM.2009.06.0701132

Li, T., Q. Ni, D. Malone and D. Leith, 2006. A new MAC scheme for very high-speed WLANs. Proceedings of the International Symposium on a World of Wireless, Mobile and Multimedia Networks, (MN' 06), IEEE Xplore Press, BuffaloNiagara Falls, New York, pp: 180-180. DOI: 10.1109/WOWMOM.2006.13
Li, T., Q. Ni, D. Malone, D. Leith and Y. Xiao et al., 2009. Aggregation with fragment retransmission for very high-speed WLANs. IEEE/ACM Trans. Netw., 17: 591-604. DOI: 10.1109/TNET.2009.2014654

Olteanu, A. and Y. Xiao, 2010. Security overhead and performance for Aggregation with Fragment Retransmission (AFR) in very high-speed wireless 802.11 LANs. IEEE Trans. Wireless Commun., 9: 218-226. DOI: 10.1109/TWC.2010.01.081291

Tinnirello, I. and G. Bianchi, 2010. Rethinking the IEEE 802.11e EDCA performance modeling methodology. IEEE/ACM Trans. Netw., 18: 540553. DOI: 10.1109/TNET.2009.2029101

Wu, Y., G. Min and L.T. Yang, 2013. Performance analysis of hybrid wireless networks under bursty and correlated traffic. IEEE Trans. Veh. Technol., 62: 449-454. DOI: 10.1109/TVT.2012.2219890

Xu, D., T. Sakurai, H.L. Vu and T. Sakurai, 2009. An access delay model for IEEE 802.11e EDCA. IEEE Trans. Mob. Comput., 8: 261-275. DOI: 10.1109/TMC.2008.108 